\title{
A Mutant of Salmonella Possessing Straight Flagella
}

\author{
By T. IINO AND MICHIKO MITANI \\ National Institute of Genetics, Misima, Japan
}

(Accepted for publication 14 April 1967)

\begin{abstract}
SUMMARY
A mutant of Salmonella typhimurium produced straight flagella in phase 2 (antigen- $I, 2$ ) and normal flagella in phase I (antigen- $i$ ). The straight flagella were observed by light microscopy and electron microscopy either with or without formalin fixation. Flagellar bundles of the mutant bacteria prepared in $0.25 \%$ methylcellulose $(\mathrm{w} / \mathrm{v})$ and examined by dark-field microscopy were also found to be straight. It was shown by electron microscopy that the component flagella of the straight flagellar bundle were in most instances irregularly twisted about each other. Heteromorphous bacteria which had straight flagella and either normal or mini-small-amplitude flagella were seen at a frequency of $10-13 \%$ among the bacterial clones in phase 2 . The bacteria with straight flagella were non-motile but they were sensitive to bacteriophage $\chi$, which is known to infect motile bacteria of Salmonella species. In transduction, using phage $P_{22}$ grown on a normal flagellar strain and the phase 2 straight strain as recipient, transductional clones with normal flagella in both phase $I$ and phase 2 were obtained. The transductional clones showed the antigen of the recipient in phase $I$ and that of the donor in phase 2 . This indicated that the straight mutant originated by a mutation of the structural gene of phase 2 flagellin. In absorption-agglutination experiments with antisera prepared against flagella of either normal- $I, 2$ or straight- 1,2 no antigenic difference between normal and straight flagella could be detected.
\end{abstract}

\section{INTRODUCTION}

Bacterial flagella are spiral filaments functioning as locomotive organelles. Their wavelength and amplitude are characteristic of each bacterial strain, and various morphological types of flagella on bacteria isolated from nature (Leifson, I960) have been described. Several types have been obtained as mutational variants of other flagellar shapes (Iino \& Mitani, 1966). Among these morphological types, the extreme one which is uncoiled has been called 'straight'. Although it had been reported that certain strains of Listeria monocytogenes (Leifson \& Palen, 1955) and Arthrobacter citruens (Leifson, I960) possessed straight flagella, Leifson (196I) showed that the straight flagellar shape of these strains was due to the formalin fixation procedure used; when this was eliminated the flagella were spirally shaped. However, one exception was a strain of Serratia indica on which small-amplitude and straight flagella were seen in unfixed preparations, while normal flagella were observed in the formalin-fixed ones (Leifson, 196I). The present report deals with a new type of mutant of Salmonella typhimurium which possesses straight flagella, whether or not fixed by formalin. 


\section{METHODS}

The Salmonella strain used in the present work was a mutant of Salmonella typhimurium TM2 (Lederberg \& Iino, 1956). The strain is diphasic, showing $i$-antigen in phase I and 1,2 -antigen in phase 2 . Mutant $\mathrm{SJ} 770$ was isolated from TM 2 by screening on semi-solid medium (Enomoto \& Iino, I963) after $N$-methyl- $N$-nitro- $N$-nitrosoguanidine treatment following method no. 3 of Eisenstark, Eisenstark \& van Sickle (1965).

Bacteriophage $\chi$, which is able to infect motile salmonellas but not paralysed or non-flagellate ones (Meynell, 196r), was received from Dr B. A. D. Stocker (Department of Medical Microbiology, Stanford University, California, U.S.A.).

Bacterial motility was observed by phase-contrast microscopy using the hangingdrop technique. Clonal motility of bacteria was examined on plates of semi-solid medium containing $0.2 \%(\mathrm{w} / \mathrm{v})$ agar and $8 \%(\mathrm{w} / \mathrm{v})$ gelatin in broth $(\mathrm{pH} 7 \cdot 2)$. For antiserum selection, semi-solid medium was supplemented with anti-H-serum, with an agglutination titre of $1 / 5000$, to a final concentration of $0.2 \%(\mathrm{v} / \mathrm{v})$.

The flagella were stained by the method of Leifson (195I). Dark-field microscopy and the general procedures of electron microscopy used were as described previously (Mitani \& Iino, 1965; Iino \& Mitani, 1966).

To decrease drying artifacts which may distort and break flagella, a method for the electron microscopy of mutant flagella was applied (suggested by D. Lang), incorporating the sedimentation of bacteria by gravity on an interface between the bacterial suspension containing cytochrome $c$ and a dense inert fluorochemical, and subsequent adsorption. Bacteria were grown in Bacto-antibiotic medium 3 (BA-3; Difco Lab., Detroit, Michigan, U.S.A.) overnight at $37^{\circ}$ without shaking, and $0.1 \mathrm{ml}$. of the bacterial culture inoculated into $5 \mathrm{ml}$. new BA-3 medium. After incubation for $3 \mathrm{hr}$ at $37^{\circ}$, the bacteria were harvested by centrifugation at $1200 \mathrm{~g}$ for $10 \mathrm{~min}$. and resuspended in $0.01 \%$ cytochrome- $c$ solution ( $\mathrm{pH} \mathrm{6.1)} \mathrm{containing} 0.25 \%$ methylcellulose. This bacterial suspension was incubated up to $20 \mathrm{hr}$ at $2-3^{\circ}$. One ml. of the bacteria + cytochrome-c mixture was added to a vessel ( $15 \mathrm{~mm}$. in diameter $\times 50 \mathrm{~mm}$. long) containing $0.7 \mathrm{ml}$. of Fluorochemical FC-42 (sp.gr. $\mathrm{I} \cdot 87 \mathrm{~g} . / \mathrm{cm}^{3}$; surface tension (air) 16 dynes/cm.; Minnesota Mining and Manufacturing Co., Saint Paul, Minnesota, U.S.A.). After $5 \mathrm{~min}$. the bacteria at the interphase were picked up by platinum grids (Siemens type) and fixed by the vapour of $2 \% \mathrm{OsO}_{4}$ for $30 \mathrm{~min}$., unless otherwise stated. Shadowing was made with platinum at an angle of $15^{\circ}$. The grids were examined in a Siemens Elmiskop $I A$ at $80 \mathrm{kV}$.

Transduction was done with phage $\mathbf{P} 22$ grown on the indicated host cultures. The general procedures of cultivation and transduction were according to the methods of Stocker, Zinder \& Lederberg (1953) and Lederberg \& Iino (1956).

Specific flagellar antisera were prepared according to Edwards \& Ewing (1955). Antigen type was identified qualitatively by slide agglutination tests with antisera having a titre of $\mathrm{I} / 100$. Quantitative measurements were made by tube agglutination tests (Kauffmann, 1954). 
RESULTS

\section{Motility of the mutant}

When organisms from a broth culture of Salmonella typhimurium TM2, mutant sJ 770, were spread on semi-solid media, both compact colonies and swarms appeared (Pl. I, fig. I). By the light microscope in a hanging-drop preparation, the bacteria from compact colonies were seen to be non-motile, while those from swarms moved translationally. Upon subcultivation both compact colonies and swarms segregated out motile bacteria and non-motile bacteria repeatedly. The frequency of the interchanges between the two types was of the order of $10^{-4} /$ bacterium/division. Examination of the flagellar antigen of these subclones showed that the change in motility corresponded exactly with the antigenic phase i.e. motile subclones were always in phase I (antigen- $i$ ) and non-motile subclones were always in phase 2 (antigen-I,2).

\section{Light microscopy of the flagella of mutant $\$ 7770$}

Phase I and phase 2 broth cultures of mutant $\mathbf{S J} 770$ were treated separately with methylcellulose $\left(0.25 \%\right.$, w/v) for $4 \mathrm{hr}$ at $23^{\circ}$ and observed by dark-field microscopy. In phase I cultures, the flagellar bundles appeared to be fuzzy straight tails on the actively moving bacteria, and to be spirally shaped with normal wavelength and amplitude in the slowly moving or stationary bacteria (Pl. I, fig. 2). Flagellar bundles in phase 2 culture were straight and not regularly coiled at all (Pl. I, fig. 3). However, these differed from the fuzzy straight tails observed in rapidly moving normal bacteria in resembling stiff rods and in being found on the stationary bacteria. The fraction of the bacteria with visible flagellar bundles increased as the time of incubation was prolonged. Comparable results were obtained in a study of stained preparations. Phase I bacteria stained by Leifson's method showed only normal flagella whether the organisms were untreated or fixed with formalin $(5 \%, \mathrm{v} / \mathrm{v}$ in broth; Pl. I, figs 4,5$)$. On the other hand, stained preparations of phase 2 bacteria showed straight flagella, whether or not the bacteria were fixed before staining by formalin (Pl. I, figs 6, 7).

\section{Electron microscopy of straight flagella}

Phase 2 organisms of mutant $\mathbf{S J} 770$ were prepared, both with and without methylcellulose, and examined in the electron microscope. Dispersed straight flagella were seen in the preparation without methylcellulose (PI. I, fig. 8). The average flagellar number per bacterium was $7 \cdot 2 \pm 0.7$, determined by examination of about 170 individual bacteria. By extensive observation of the flagellated bacteria, it was found that $10-13 \%$ were heteromorphous with regard to their flagellar morphology. These heteromorphous bacteria, besides straight flagella, possessed either normal or 'minismall-amplitude' flagella. The term mini-small-amplitude is proposed to describe a new flagellar shape in which the wavelength and amplitude are $0.78-\mathrm{I} \cdot 9 \mu$ and $0.08-0 \cdot \mathrm{I} \mu$, respectively. The overall shape of mini-small-amplitude flagella is analogous to that of the small-amplitude type (Iino \& Mitani, 1966), except that the wavelength is approximately one-third of that of the small-amplitude type. Within a clone, heteromorphous bacteria which carried both straight and normal or straight and mini-smallamplitude flagella were usually observed at the same frequency. The number of normal or mini-small-amplitude flagella in these clones was less than three per bacterium 
(Pl 2, fig. 9). Less frequently (in about $2 \%$ of the organisms), heteromorphous bacteria which carried straight, normal and mini-small-amplitude flagella were seen. Furthermore, at a frequency of about $\mathrm{I}$ in 500 , single flagella were observed which were heteromorphous, themselves possessing either straight and mini-small-amplitude or straight and normal regions.

Flagellar shape was observed after 3 and $20 \mathrm{hr}$ of treatment of the bacteria with $0.25 \%$ methylcellulose. In the former preparation, flagellar bundles were hardly seen, while in the latter $94.7 \%$ of the bacteria formed flagellar bundles. The overall shape of the bundle was not helical but straight, comparable to the observations by dark-field microscopy. The component flagella of the bundle were irregularly twisted at different intervals in bundle to bundle (P1. 2, figs IO, II).

\section{Stability of the mutant $\$ 9770$}

Because of the immobility of bacteria with straight flagella, revertants which recovered motility in phase 2 could be selected from the bacterial population of mutant SJ 770 as swarms on semisolid agar plates containing phase I antiserum. When $1.5 \times 10^{7}$ bacteria from a single colony of mutant $\mathrm{SJ} 770$ in phase 2 were spread on semisolid agar and incubated at $37^{\circ}$ for $48 \mathrm{hr}$, no swarms were detected in 20 independent experiments. When the innoculum was increased to $5 \times 10^{8}$ bacteria, one to five swarms were detected in each experiment. Fifteen such swarms, selected in independent experiments, were transferred to broth and the motility and flagellar shape of the component bacteria were examined. It was found that these reverted clones consisted of actively motile bacteria with flagella of normal wavelength and amplitude.

\section{Genetic analysis of the straight flagellar mutant}

Transduction was carried out using a phase I culture of mutant $\mathbf{S J} 770$ (normal- $i$ : straight- $I, 2)$ as recipient and phage prepared on a phase 2 culture of Salmonella

Table I. Transduction of a phase 2 culture of Salmonella strain SL 688 (b:e,n,x) with a phase I culture of $S$. typhimurium $5 \Im 770$ (i: straight-I,2)

Recombinants were selected on plates of semisolid agar medium containing anti- $i$ serum. Donor: $3 \times 10^{9}$ phage particles; recipient: $1 \times 10^{9}$ bacteria.

\begin{tabular}{|c|c|c|c|c|c|}
\hline & Flage & antigens & Flag & pe in & \\
\hline & Phase I & Phase 2 & Phase I & Phase 2 & clones \\
\hline Donor & $b$ & $e, n, x$ & Normal & Normal & - \\
\hline Recipient & $i$ & $I, 2$ & Normal & Straight & - \\
\hline Recombinants & $i$ & $e, n, x$ & Normal & Normal & $3 \mathrm{I}$ \\
\hline & $b$ & $I, 2$ & Normal & Straight & 36 \\
\hline
\end{tabular}

strain SL688, which produces normal flagella in both phase I (antigen- $b$ ) and phase 2 (antigen-e,n,x) (Iino \& Enomoto, I966). The use of phase I bacteria as recipient has two advantages: first, both phase $I$ and phase 2 antigen type recombinants should be detectable, and secondly, even if genotypically normal- 1,2 type clones appear by reversion, the $I, 2$ antigens will not be expressed unless phase variation from phase I to phase 2 occurs simultaneously (Lederberg \& Iino, 1956).

A mixture of $3 \times 10^{9}$ phage particles propagated from the donor and $\mathrm{I} \times 10^{9}$ recipient bacteria were spread on semisolid agar plates containing anti- $i$ serum. Transductional 
clones identified as swarms on the plates were isolated, and their antigen type and the shape of their flagella examined. The results are listed in Table I. Among 67 clones examined, 36 possessed the phase $\mathrm{I}$ antigen of the donor $b$, but in phase 2 they showed the recipient type antigen, $I, 2$. Their flagellar shapes were normal in phase $I$ and straight in phase 2 . The remaining 3 I clones possessed the phase 2 antigen of the donor, $e, n, x$, and the phase $\mathrm{I}$ antigen of the recipient, $i$. Their flagellar shapes were normal in both phase $I$ and phase 2 . These results indicate that the straight flagellar character in phase 2 of mutant 53770 is replaced by the normal when the phase 2 antigen type determinant of the normal strain is transduced into the straight flagellar mutant.

To examine the possibility of the rare occurrence of normal-I,2 type recombinants, the transduction was repeated on a larger scale on semisolid agar plates containing $b, i$, and $e, n, x$ antisera. A transduction mixture containing $3 \times 10^{10}$ phage particles propagated in the donor and $\mathrm{I} \times 1 \mathrm{IO}^{10}$ recipient bacteria was spread on the plates. In this experiment, in which approximately 300 normal-e, $n, x$ recombinants per plate were anticipated, the appearance of swarms, which would indicate the occurrence of normal-I,2 type recombinants, was not detected. Thus, as far as has been tested, the mutant site of the straight-flagella character was inseparable from the determinant of phase 2 flagellar antigen by recombination.

\section{Flagellar antigens of the mutant}

The $\mathrm{H}$-antigen of the straight flagellar mutant was identified as $I, 2$ by slideagglutination tests. For detailed comparison of the $\mathrm{H}$-antigen of normal and straight flagella, anti-I,2 sera were obtained against Salmonella typhimurium TM 2 and its straight flagellar mutant $\$ \mathbf{3} 770$. The agglutination titres of the anti- $I, 2$ sera determined with both $I, 2$ type bacteria were examined before and after reciprocal absorption. The titres of the antisera before absorption were $2^{14}$ in both antisera. After cross-absorption, no agglutination was observable over the range of dilutions of $1 / 2^{3}$ to $1 / 2^{15}$. Therefore, the $I, 2$ antigens of TM 2 and its straight flagellar mutant $\mathrm{SJ} 770$ show identity in the absorption-agglutination test.

Sensitivity of the straight flagellar mutant to bacteriophage $\chi$

A $\chi$-phage suspension propagated on a broth culture of Salmonella typhimurium TM 2 and having a titre of $10^{8}$ particles $/ \mathrm{ml}$. was diluted sequentially tenfold down to

Table 2. Efficiency of plating of bacteriophage $\chi$ on normal, straight and paralysed flagellar strains of Salmonella typhimurium

\begin{tabular}{|c|c|c|c|c|}
\hline $\begin{array}{l}\text { S. typhimurium } \\
\text { strain }\end{array}$ & $\begin{array}{l}\mathrm{H} \text {-antigen } \\
\text { type }\end{array}$ & $\begin{array}{l}\text { Flagellar } \\
\text { character }\end{array}$ & EOP* & SD + \\
\hline TM 2 & $I, 2$ & Normal & $1 \cdot \infty$ & - \\
\hline SJ 770 & $i$ & Normal & $0.93 \pm$ & 0.09 \\
\hline SI 770 & 1,2 & Straight & $0.95 \pm$ & 0.10 \\
\hline SJ 60 & 1,2 & Paralysed & 0.00 & - \\
\hline
\end{tabular}

$\mathrm{ro}^{2}$ particles $/ \mathrm{ml}$. Each of the dilutions was mixed with an equal volume of a broth culture ( $10^{7}$ bacteria/ml.) of TM2-normal- $-2,2$, SJ 770-normal- $i$, SJ 770 -straight- $I, 2$ or su 60-I, 2, a paralysed mutant ( $\mathrm{mot}^{-}$) of TM 2 (Enomoto, I966) and $0 \cdot \mathrm{I} \mathrm{ml}$. of each mixture 
was spread on a $0.6 \%(\mathrm{w} / \mathrm{v})$ nutrient agar plate. After incubation for $\mathrm{Io} \mathrm{hr}$ at $37^{\circ}$, the number and morphology of the developed plaques were examined. In three independent experiments, plaques were not detected at all on the plates of sJ60. However, when the phages were plated on TM 2-normal- 1,2 , sJ 770-normal- $i$ and sJ 770 -straight- $I, 2$ the plaques appeared with $100 \%$ efficiency in all three strains (Table 2). The size and morphology of the plaques were also indistinguishable among the three: they were clear and circular, having a diameter of $0.5-1.5 \mathrm{~mm}$.

\section{DISCUSSION}

The straight mutant flagella described here differ from those reported previously (Leifson \& Palen, 1955; Leifson, 1960, 1961) in that they are straight whether or not they have undergone formalin fixation. Dark-field microscopy and electron microscopy provided additional support for the idea that the straight flagella character observed by light microscopy of the stained materials is not an artifact caused by stretching of the spiral but is an intrinsic property of the mutant.

One characteristic of the mutant is that the straight flagella appear only in phase 2. Transductional analysis further demonstrated that the mutant site of the straight flagella is inseparable by recombination from the phase 2 antigen determinant. From the accumulated data (Iino, 1962; McDonough, 1965) it appears that the specificity of the flagellar antigen in each phase is determined by the structural gene of flagellin, the component protein of flagella, i.e. $H_{I}$ in phase $I$ and $H_{2}$ in phase 2. Other flagellar shape mutants which have been studied in Salmonella were all found to express their mutant characters only in phase $I$ or phase 2 , and their mutant sites were located in the structural gene of flagellin of the corresponding phase (Iino, 1962; Iino \& Mitani, 1966).

Differences in the tryptic peptide maps of flagellin between the flagellin of a curly flagellar mutant and that of the parental wild-type strain have been found (Enomoto \& Iino, 1966). Although chemical analysis has not been done with the flagellin on the straight flagella, it is reasonable to infer that the straight flagellar characteristic is due to mutation of the structural gene of phase 2 flagellin, resulting in a change in the primary structure of the flagellin of the mutant. This difference in primary structure is manifested in the conformation of the flagellin molecule itself and in the mode of polymerization of the flagellins to flagellar fibres. The immunological identity of the $\mathrm{H}$-antigens of the normal strain and the flagellar shape mutants, and the absence of immunological cross-reactions between flagella with identical shapes indicate that the determinants of the $\mathrm{H}$-antigen and of the characteristic flagellar shape are present at different sites in the flagellin molecule.

A characteristic feature of the straight flagellar mutant is the heteromorphism of flagella on a single bacterium, i.e. one organism with normal or mini-small-amplitude flagella and straight ones in a phase 2 clone. The frequency of this heteromorphism varied from clone to clone in the range of IO-13\%. Moreover, bacteria possessing only mini-small-amplitude or normal flagella were not detected. Mini-small-amplitude flagella cannot be defined as a particular shape because some intermediate flagella from straight to more or less mini-small-amplitude were seen. It is very difficult to determine whether the straight mutant produces mini-small-amplitude flagella together with straight or irregular ones, because of possible artifacts appearing during 
preparation for electron microscopy. Bacteria with only normal flagella were seen only one-tenth as often as straight-normal heteromorphous bacteria; the former are presumed to occur by phase variation from phase 2 to phase I (Lederberg \& Iino, 1956). The frequent appearance of heteromorphous bacteria observed in phase 2 clones of mutant $\mathrm{SJ} 770$ is hardly attributable to flagellar phase variation or mutation. Together with the flagellar heteromorphism observed in the heteromorphous mutant in Salmonella abortus-equi (Iino \& Mitani, I966), the phenomenon offers an interesting problem concerning the morphological differentiation of cellular organelles. A straight flagellar strain of Serratia indica reported by Leifson (196I) is also heteromorphous: it produces both straight and small-amplitude flagella.

Changes in the spiral shape of flagella by mutation are very often associated with changes in the mode of cellular movement (Iino \& Mitani, I966). This is most dramatic in the straight flagellar mutant; in liquid medium the mutant bacteria are completely non-motile. The formation of a flagellar bundle during methylcellulose treatment does not effectively restore mobility to the mutant bacterium. This indicates that the spiral structure of flagella is essential for the effective movement of the flagellated bacteria.

Bacteriophage $\chi$ has been known to be able to infect salmonellas when the bacteria are flagellated and motile. It cannot infect paralysed strains which harbour mutations at the mot loci even though they do have flagella structurally indistinguishable from those of the parental motile strain (Meynell, 1961). Although the straight flagellar bacteria are non-motile in liquid media, their response to $\chi$-phage is quite different from the paralysed mutant bacteria in that they have retained the same degree of sensitivity to $\chi$-phage as normal bacteria. This may be explained by assuming that cellular locomotion per se is not a prerequisite for infection by $\chi$-phage but rather that the presence of motile flagella is. Thus, in the straight flagellar mutants, the flagella per se may have retained motility but, because of loss of the spiral structure, this movement may not be capable of effecting locomotion of the bacterial bodies.

Contribution from the National Institute of Genetics, Japan, no. 650. This work was supported by Public Health Service Research Grant (AI-02872) from the National Institute of Allergy and Infectious Diseases, U.S.A., and by a grant from the Tokyo Rayon Foundation for the promotion of Science and Technology. The electron microscopy was done after the second author moved to the present address. We are thankful to Dr Dimitrij Lang at the Southwest Center for Advanced Studies for advice concerning the electron microscopy and for partial financial support. We are also grateful to Dr Ronald Bauerle and Mrs Moreen Higgs for revision and typing of the manuscript.

\section{REFERENCES}

EDWARDs, P. R. \& EWING, W. H. (1955). Identification of Enterobacteriaceae. Minneapolis, Minn., U.S.A.: Burgess Publ. Co.

Eisenstark, A., Eisenstark, R. \& van Sickle, R. (1965). Mutation of Salmonella typhimurium by nitrosoguanidine. Mutation Res. 2, 1.

Enomoto, M. (1966). Genetical studies of paralyzed mutants in Salmonella. I. Genetic fine structure of the mot loci in Salmonella typhimurium. Genetics 54, 715.

ENomoto, M. \& InNo, T. (I963). Colonial dimorphism in nonmotile Salmonella. J. Bact. 86, 473.

ENomoto, M. \& InNo, T. (1966). The comparison of normal and curly flagella in Salmonella abortusequi by two dimensional separation of peptides. Jap. J. Genet. 4I, 131 .

IINo, T. (1962). Curly flagellar mutants in Salmonella. J. gen. Microbiol. 27, 167. 
Ino, T. \& ENomoto, M. (1966). Genetical studies of non-flagellate mutants of Salmonella. J. gen. Microbiol. 43, 315.

IINo, T. \& Mitani, M. (1966). Flagellar shape mutants in Salmonella. J. gen. Microbiol. 44, 27.

Kauffmann, F. (1954). Enterobacteriaceae, 2nd ed. Copenhagen, Denmark: E. Munksgaard.

Lederberg, J. \& IINO, T. (1956). Phase variation in Salmonella. Genetics 4I, 743.

LEIFSON, E. (I95I). Staining, shape and arrangement of bacterial flagella. J. Bact. 62, 377.

LeIrSON, E. (1960). Atlas of Bacterial Flagellation. New York: Academic Press.

LEIFSON, E. (1961). The effect of formaldehyde on the shape of bacterial flagella. J. gen. Microbiol. 25, I3I.

Leifson, E. \& Palen, M. I. (1955). Variation and spontaneous mutation in the genus Listeria in respect to flagellation and motility. J. Bact. 7o, 233.

McDonough, M. W. (1965). Amino acid composition of antigenically distinct Salmonella flagellar proteins. J. molec. Biol. 12, 342.

MeYNeLL, E. W. (I96I). A phage, $\chi$, which attacks motile bacteria. J. gen. Microbiol. $25,253$.

MITANI, M. \& IINO, T. (1965). Electron microscopy of bundled flagella of the curly mutant of Salmonella abortivoequina. J. Bact. 90, 1096.

STOCKER, B. A. D., ZINDER, N. D. \& LEDERBERG, J. (I953). Transduction of flagellar characters in Salmonella. J. gen. Microbiol. 9, 410.

\section{EXPLANATION OF PLATES}

\section{Plate I}

Fig. I. A broth culture of Salmonella typhimurium mutant $s J 770$ was diluted and about 400 organisms/ $0.1 \mathrm{ml}$. were spread on a semisolid agar and incubated for $10 \mathrm{hr}$ at $37^{\circ}$. Swarms and compact colonies appeared on the plate. Swarms correspond to phase 2 clones and compact colonies to phase $\mathrm{I}, \times 0.6$. Figs 2, 3. Dark-field micrographs of the organisms of mutant su 770 . In fig. 2 a normal flagellar bundle of a phase 1 organism and in fig. 3 straight flagellar bundle of a phase 2 organism are seen. $\times 4000$.

Figs 4-7. Organisms of mutant su 770 whose flagella were stained by Leifson's method. In figs 4,5 are shown phase 1 organisms with normal flagella, and figs 6,7 phase 2 organisms with straight flagella. Figs 4, 6 are unfixed preparations, and figs 5,7 are formalin-fixed $\times 1000$.

Fig. 8. Electron micrographs of a phase 2 organism of mutant $\mathbf{5} 770$. Organisms were negatively stained with $\mathrm{r} \%$ bovine serum albumin $\left(\mathrm{pH}_{7} \cdot 3\right) . \times 10,400$.

\section{Plate 2}

Fig. 9. Electron micrograph of a heteromorphous organism present in phase 2 of mutant $5 J 770$ prepared by shadowing. $\times 13,000$. Straight flagella and a typical mini-small-amplitude flagellum are seen attached to single organism.

Figs 10, 11. Electron micrographs of phase 2 organisms of mutant sJ 770 incubated with $0.25 \%$ methylcellulose for $20 \mathrm{hr}$ at $2^{\circ}-3^{\circ}$ and prepared by shadowing. The figures show bundles of straight flagella which are irregularly twisted together at different intervals. 

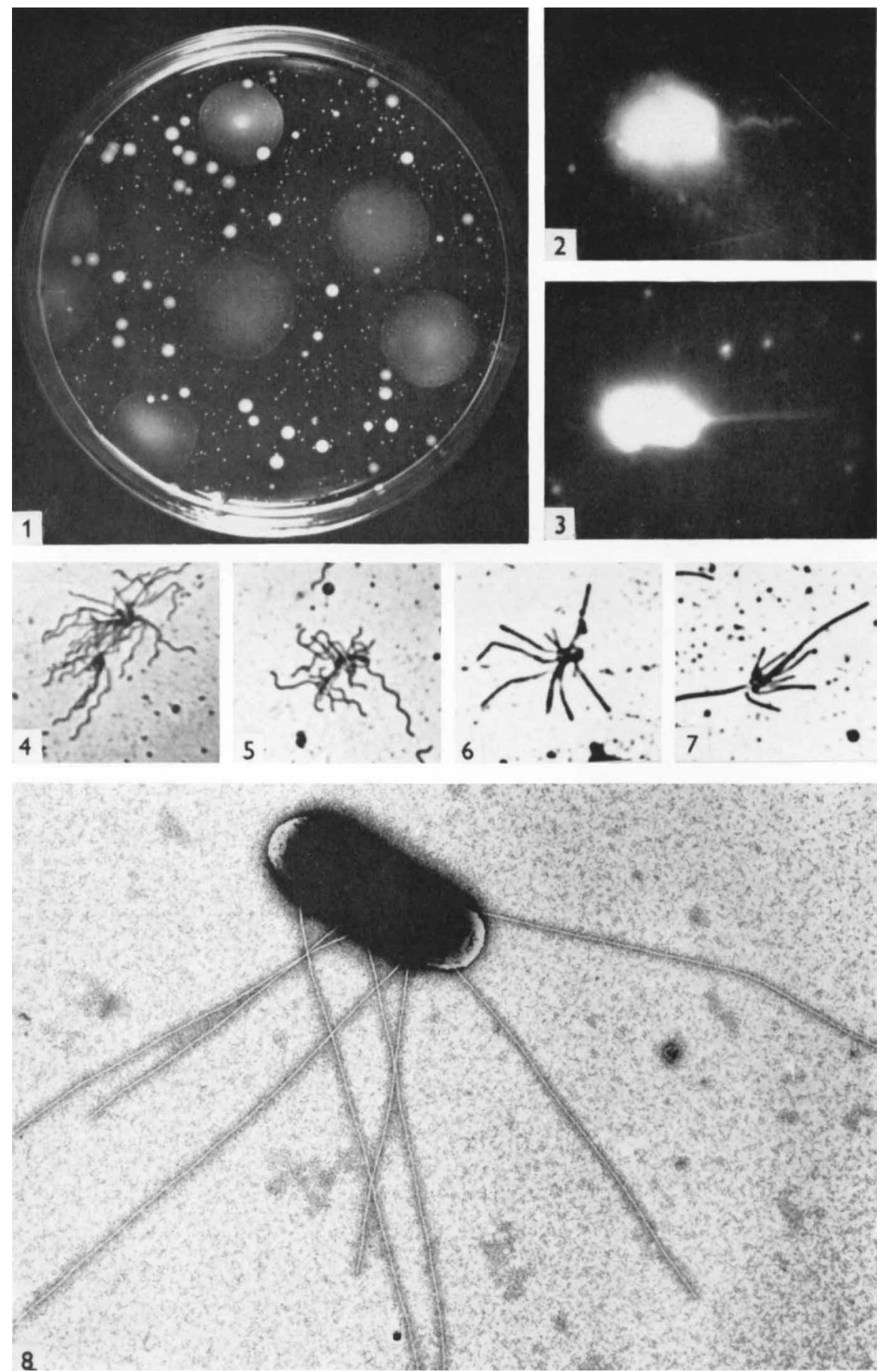

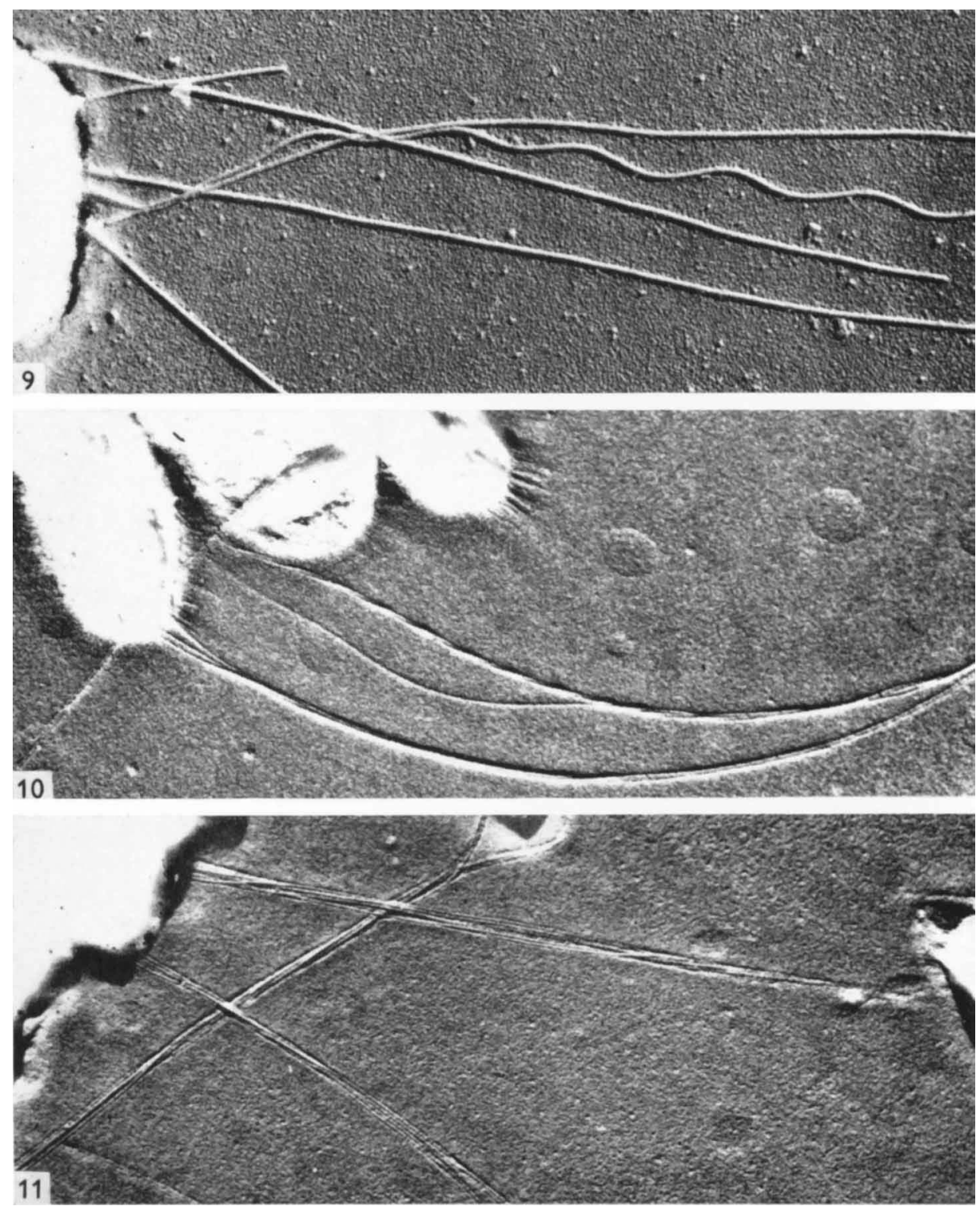